\title{
Maternidad en adolescentes en una población de refugiados
}

\author{
Milivoj Jovančević, MD, pediatrician \\ Mladen Knežević, sociologist, University \\ professor \\ Branka Makalouš-Smuđ, MD, School \\ medicine specialist \\ Asociación Psiquiátrica Croata \\ CROACIA
}

\section{Introducción}

\section{Maternidad en la adolescencia}

La cuestión principal del problema de la maternidad adolescente surgió de la experiencia de adolescentes que habían tenido que crecer en los aspectos biológicos, cognitivos, psicológicos y sociales, mientras estaban sometidas a un estrés crónico marcado particularmente por la pobreza, por limitaciones para obtener escolarización y por el problema de la inestabilidad familiar. La mayoría de estas madres adolescentes sufrieron problemas psicológicos, sociales y económicos, permanentes o durante un tiempo.

Džepina et al. (1990) investigaron una población de adolescentes croatas, documentando que más de la mitad de las participantes no usaban medidas de protección en sus relaciones sexuales, por lo que un $4,5 \%$ de las adolescentes sexualmente activas estaban embarazadas y, de ellas, la quinta parte dieron a luz (Džepina y Štampar 1990, Štampar y Beluhan 1991). En comparación con las madres adultas, Osoffsky y Hann señalaron 
que la identidad de las madres adolescentes era más dispersa, eran más dependientes, se encontraban más a menudo deprimidas, con menor auto-respeto, y tuvieron dificultades para establecer confianza en sus relaciones (Osofsky et al. 1992).

Algunos indicadores fiables y significativos presentaron a las madres deprimidas como emocionalmente más distantes, no se dedicaban a sus hijos de manera normal y muchas de ellas tenían tendencia a un mayor riesgo de agresión física hacia sus propios hijos (Zuravin 1989). Todo ello ciertamente implicó un mayor riesgo para los niños, de verse afectados por problemas emocionales (Garrison et al. 1989, Osofsky 1991). La pobreza y la indigencia contribuyeron de modo importante al problema que debía afrontar la madre adolescente: incrementó el riesgo de vivir bajo circunstancias difíciles, derivando en ocasiones en una vida al borde de la ley, cambios frecuentes de domicilio, problemas para llevar a cabo las obligaciones diarias, dificultades para criar a los niños y carencias de apoyo emocional y social. Muchas madres adolescentes hablan poco con sus hijos y se podría prever una inadecuada habilidad del niño para comunicarse. Muchos estudios han presentado a las madres adolescentes como poco conocedoras de las necesidades de sus hijos y con poca habilidad para guiarlos en la vida, en comparación con madres adultas (Furstenberg et al. 1987).

Parece, sin embargo, que a pesar de los problemas de la maternidad adolescente, algunas madres jóvenes y sus hijos progresan suficientemente bien. Ha habido muchas razones para una maternidad adolescente satisfactoria $\mathrm{y}$, ciertamente, una clave es el apoyo dado a las madres jóvenes por su familia, sus amigos $\mathrm{u}$ otro tipo de apoyos, el hecho de recibir ayuda adecuada y cuidado alternativo de otra persona en casa, y también la posibilidad de continuar su escolarización, así como evitar embarazos posteriores (Furstenberg et al.
1987). El auto-respeto es el siguiente elemento, muy importante para el éxito en la maternidad adolescente. Cuanto mejor se sienta una madre consigo misma, más afectiva será con su hijo. Hay evidencia de una maternidad más problemática en madres muy deprimidas con bajo nivel de auto-respeto (Osofsky et al. 1988, Achenbach 1979).

A pesar de las diferencias teóricas que intentan perfilar la relación padres-hijos, se coincide en la sugerencia de que el componente principal para el éxito de la maternidad es la sensibilidad de los padres. La conducta emocional de los padres implica una habilidad para desarrollar una respuesta adecuada a la señal del hijo, en particular a la más importante, el llanto del niño. La madre tiene que observar la necesidad del hijo, interpretarla correctamente y responder a la misma de forma efectiva. Las parejas maduras con niños consiguieron solucionar ampliamente los problemas que estaban relacionados, en primer lugar, con la edad de la adolescente y los acontecimientos estresantes que debía afrontar la persona joven en este contexto. Por ello, la habilidad de una adolescente para ser una buena madre ha sido objeto de muchos estudios. Las adolescentes, en el pico de su desarrollo físico, psicológico y social, son a veces un problema para sí mismos, de tipo psicosocial la mayoría, y estas personas podrían tener disminuidas las habilidades para una maternidad sensible, generando así consecuencias a largo plazo para el crecimiento del niño.

La guerra trae consigo varios factores negativos que trastornan una maternidad normal y el desarrollo psicológico y emocional del niño. La pobreza, las relaciones rotas de familia (pérdidas en la familia nuclear), la adolescencia, el miedo existencial, la vida en los centros colectivos, todos estos factores afectan simultáneamente la habilidad para desarrollar los finos hilos necesarios para una maternidad exitosa. 


\section{Muestra}

Examinamos grupos de 121 madres adolescentes menores de 19 años, que habían sido acomodadas junto a sus hijos en campos de refugiados y centros colectivos en Croacia y Bosnia-Herzegovina durante 1995/96 y 97.

Como se indica en la Tabla I, 707 madres habían sido objeto de experiencias traumáticas; como media, cada madre experimentó 4,13 acontecimientos traumáticos. Sólo 38 no sufrieron trauma, 5 adolescentes $(4,3 \%$ del total de adolescentes) y 33 madres no adolescentes $(5,29 \%)$. Por lo que respecta a las estadísticas, no hubo diferencias entre ninguno de los grupos, ya que las madres de ambos compartieron las mismas experiencias. A pesar de no haber diferencias estadísticamente signifi- cativas entre los grupos, nos gustaría señalar que las madres adolescentes habían pasado, en porcentaje, por más experiencias traumáticas, condicionadas por razones personales (pérdida de parientes de primer grado, haber sido testigos de violencia o muerte de otras personas), mientras que las madres no adolescentes fueron objeto de más traumas, relacionados con la pérdida de propiedades o de otros valores, pérdida de empleo y maridos en el frente; todo ello dependiendo, por supuesto, del grupo de edad de las adolescentes, así como del estatus social.

En el grupo control examinamos 624 madres adultas y sus hijos (madres no adolescentes), que vivían bajo las mismas condiciones en los siguientes centros colectivos: Gašinci, Čepin, Rokovici, Grude, Posušje,

Tabla I

Experiencias traumáticas

Acontecimiento traumático

\begin{tabular}{rrrr}
$\begin{array}{c}\text { Madres } \\
\text { adolescentes }\end{array}$ & \multicolumn{3}{c}{$\begin{array}{c}\text { Otras } \\
\text { madres }\end{array}$} \\
\hline 192 & 30,77 & 39 & 32,23 \\
22 & 3,53 & 3 & 2,48 \\
& & & \\
253 & 40,54 & 47 & 38,84 \\
263 & 42,15 & 43 & 35,54 \\
297 & 47,60 & 53 & 43,80 \\
224 & 35,90 & 32 & 26,45 \\
198 & 31,73 & 32 & 26,45 \\
488 & 78,21 & 91 & 75,21 \\
12 & 1,92 & 1 & 0,83 \\
3 & 0,48 & 0 & 0 \\
& & & \\
13 & 2,08 & 4 & 3,31 \\
47 & 7,53 & 3 & 2,48 \\
12 & 1,92 & 3 & 2,48 \\
85 & 13,62 & 20 & 16,53 \\
111 & 17,79 & 25 & 20,66 \\
3 & 0,48 & 0 & 0 \\
0 & 0 & 0 & 0 \\
329 & 52,72 & 67 & 55,37 \\
91 & 14,58 & 9 & 7,44 \\
& & & \\
24 & 3,85 & 3 & 2,48 \\
25 & 4,01 & 2 & 1,65 \\
33 & 5,29 & 5 & 4,13 \\
624 & & 121 & \\
\hline & & &
\end{tabular}

1. Muerte de un ser querido

2. Resultar herido o sufrir una enfermedad grave

3. Resultar herido o que un miembro de la familia sufra una enfermedad grave

4. Pérdida o daño considerable de posesiones personales

5. Pérdida o daño considerable de otras posesiones

6. Pérdida de pertenencias con gran valor sentimental

7. Pérdida de la oportunidad para hacer dinero para vivir

8 Miembros de la familia luchando en el frente

9. Separación de niños pequeños (debido al exilio)

10. Separación de otros niños (debido al exilio)

11. Separación de los padres debido al exilio (para niños mayores de 18 años)

12. Separación del cónyuge (debido al exilio)

13. Prisión (de más de siete días)

14. Ser testigo de violencia

15. Ser testigo de la muerte de otras personas

16. Ser víctima de violación o acoso sexual

17. Ser testigo de violación

18. Exposición (directa) a ataques enemigos

19. Pasar hambre durante un largo periodo de tiempo

20. Exposición a condiciones climáticas adversas durante un largo periodo de tiempo

21. Ser interrogado por las fuerzas enemigas

22. Ninguna experiencia traumática Total 
Neum, Ljubuški, Tomislavgrad, Čapljina y la región de Bihać.

\section{Método}

A través del contacto diario con las madres jóvenes durante su embarazo, parto y aceptación del hijo, tenemos una visión del entorno psicológico en la familia, y pudimos ver los síntomas del trauma y los problemas en la adpatación a las condiciones de refugiado y a una forma de vida totalmente diferente; supervisamos el progreso de los niños y, en particular, observamos la relación de las madres con ellos. Los equipos estaban formados por un psicólogo / psiquiatra infantil, una enfermera titulada en pediatría y un pediatra, y llevaron a cabo el estudio empleando una entrevista estructurada y una lista protocolizada para la recogida de datos.

\section{Resultados}

Como se esperaba, hay una diferencia significativa entre los dos grupos. Se pueden dis- tinguir, de manera estadísticamente significativa, dos tipos de comunidades. En primer lugar, la mayoría de las madres no adolescentes estaban casadas y vivían con su marido, a diferencia de las madres adolescentes. Sin embargo, la mayoría de las madres adolescentes vivían con sus parejas de hecho. Ésta fue una elección comprensible, debido al ambiente de guerra. De hecho, las madres menores de 18 años deberían haber obtenido permiso judicial para casarse de acuerdo con el Derecho de Familia en la ex-Yugoslavia.

Los datos obtenidos indican una discrepancia significativa en el origen de las madres, relacionado con sus condiciones de vida anteriores a la guerra. Las madres adolescentes generalmente venían de las áreas más empobrecidas $\left(\mathrm{Chi}^{2}=5,54 ; \mathrm{p}=0,05\right)$. Incluso antes de la guerra, la maternidad adolescente en tales áreas no era infrecuente. Las madres no adolescentes provenían principalmente de áreas urbanas $\left(\mathrm{Chi}^{2}=5,62 ; \mathrm{p}=0,05\right)$. $\mathrm{La}$ situación inadecuada de maternidad adolescente empeoró por el conflicto de culturas. Una gran parte de las madres adolescentes tuvieron que vivir como refugiadas en áreas urbanas donde, al mismo tiempo, tuvieron que readaptarse dramáticamente al nuevo entorno social: provenientes de pequeños

Tabla II

Estado civil de las mujeres evaluadas

\begin{tabular}{|c|c|c|c|c|c|}
\hline Estado civil & Grupo & $\mathrm{N}$ & $\%$ & $\mathrm{Chi}^{2}$ & I.C. \\
\hline \multirow[t]{2}{*}{ Casada } & Otras madres & 510 & 81,73 & 23,44 & $95 \%$ \\
\hline & Madres adolescentes & 75 & 61,98 & & \\
\hline \multirow[t]{2}{*}{ No casada, pero viviendo en pareja } & Otras madres & 65 & 10,42 & 29,88 & $95 \%$ \\
\hline & Madres adolescentes & 35 & 28,93 & & \\
\hline \multirow[t]{2}{*}{ Ni casada ni viviendo en pareja } & Otras madres & 16 & 2,56 & 0,21 & $95 \%$ \\
\hline & Madres adolescentes & 4 & 3,31 & & \\
\hline \multirow[t]{2}{*}{ Viuda, divorciada } & Otras madres & 12 & 1,92 & 0,4 & $95 \%$ \\
\hline & Madres adolescentes & 2 & 1,65 & & \\
\hline \multirow[t]{2}{*}{ Desconocido } & Otras madres & 21 & 3,37 & 0,18 & $95 \%$ \\
\hline & Madres adolescentes & 5 & 4,13 & & \\
\hline \multirow[t]{2}{*}{ Total } & Otras madres & 624 & & & \\
\hline & Madres adolescentes & 121 & & & \\
\hline
\end{tabular}


pueblos atrasados, se instalaron en una ciudad donde vivían como refugiadas y tenían que afrontar las dificultades de una maternidad inapropiada. Si tal maternidad había sido socialmente aceptada en el área de la que venían originalmente, en el nuevo entorno dicha maternidad se consideró inapropiada.

También intentamos comparar los problemas de escolarización en el grupo de padres no adolescentes. Sin embargo, las diferencias entre los padres y las madres sugirieron que estaban implicados tanto algunos patrones culturales de la población como el grado de desarrollo de la misma. De hecho, los maridos tenían significativamente mayor nivel de escolarización que sus esposas, especialmente entre las madres no adolescentes.

Un mayor número de madres adolescentes, en relación a sus parejas, interrumpieron su educación primaria, de manera estadísticamente significativa. La diferencia fue muy sorprendente respecto a la educación secundaria incompleta, donde las madres adolescentes interrumpieron sus estudios más frecuentemente que sus parejas. Por lo que respecta a la finalización satisfactoria de la educación secundaria, las parejas de las madres adolescentes tuvieron mucho más éxito que las mismas madres. Sólo alrededor de un 13,22\% de las madres adolescentes consiguieron completar los estudios de secundaria, mientras que el 32,23\% de sus parejas tuvieron más éxito y finalizaron el mismo nivel de educación $\left(\mathrm{Chi}^{2}=12,45\right.$; $\mathrm{p}=0,05)$.

Un problema especial para las madres fue el trabajo de la pareja, considerado como un importante indicador socio-económico. Puesto que, en esta cultura, la figura del padre representaba la piedra angular de la estabilidad familiar, los datos mostrados no sólo reflejan una característica socio-económica, sino que también se derivan implicaciones psicológicas importantes para ambos compañeros y para sus hijos. Hubo diferencias estadísticamente significativas en el trabajo de los padres, parejas de las madres adolescentes. Nuestro trabajo documentó una amplia diferencia en la posición socio-económica de los dos grupos examinados. Casi el 60\% de las familias adolescentes no tenían ni trabajo ni ingresos, frente a, aproximadamente, el $40 \%$ de las familias de madres no-adolescentes. Sólo el 15,70\% de las parejas de las madres adolescentes tenían una fuente de ingresos regular. Sería lógico, pues, extraer la siguiente conclusión: los dos grupos examinados pertenecían a grupos de edad crucialmente diferentes; los padres-parejas de las madres adolescentes eran, naturalmente, más jóvenes.

Obviamente, este hecho no facilitó la posición de la familia de la madre adolescente sino que, por el contrario, la hizo mucho más dura. En una situación donde el empleo era considerado como un privilegio excepcional,

Tabla III

Periodo de embarazo*

\begin{tabular}{llrrrr} 
Variable & \multicolumn{1}{c}{ Grupo } & $\mathrm{n}$ & $\%$ & $\mathrm{Chi}^{2}$ & I.C. \\
\hline Examen ginecológico: yo no lo necesitaba & Otras madres & $\mathbf{2 9}$ & $\mathbf{4 , 6 5}$ & $\mathbf{7 , 0 8}$ & $\mathbf{9 9 \%}$ \\
& Madres adolescentes & $\mathbf{1 3}$ & $\mathbf{1 0 , 7}$ & & \\
Complicaciones en el embarazo & Otras madres & $\mathbf{7 5}$ & $\mathbf{1 2 , 0 2}$ & $\mathbf{4 , 0 2}$ & $\mathbf{9 5 \%}$ \\
& Madres adolescentes & $\mathbf{7}$ & $\mathbf{5 , 7 9}$ & & \\
Complicaciones en el parto & Otras madres & 75 & 12,02 & 0,85 & $95 \%$ \\
& Madres adolescentes & 11 & 9,07 & & \\
\hline
\end{tabular}

* Las filas en negrita representan diferencias significativas. 
las familias de las madres adolescentes experimentaban una pérdida de privilegios.

Casi todas nuestras madres jóvenes dejaron el colegio durante el embarazo. Un 17,3\% tuvo su segundo bebé a la edad de 19 años. La mayoría de ellas no tuvieron complicaciones, y dieron a luz a los bebés a término, con una adecuada asistencia en un hospital. Las complicaciones del embarazo fueron significativamente más frecuentes en el grupo de las madres adultas (no adolescentes).

La desatención de los niños en el grupo examinado ya se había iniciado, de alguna manera, antes del nacimiento, puesto que los datos indican que el $10,7 \%$ de las madres adolescentes no pasó una revisión ginecológica, a pesar de que tuvieran oportunidad para ello. Las madres adolescentes intentaron justificar su descuido sugiriendo que no había necesidad, o diciendo que estos exámenes eran inusuales.

Observando las necesidades de adaptación a las extremas condiciones de vida en los centros de refugiados, aprendimos que las madres jóvenes tenían más problemas a la hora de ser independientes, y que eran significativamente menos colaboradoras con los grupos de expertos, en comparación con las madres adultas (no adolescentes). El trastorno de adaptación, diagnosticado mediante evaluación psiquiátrica, fue un problema importante en las madres adolescentes, y estaba causado por la adolescencia, el embarazo y la experiencia de una guerra. La depresión fue, sin embargo, sig-

Tabla IV

Problemas emocionales*

Variable

Grupo $\mathrm{n}$

Trastorno de adaptación

Otras madres

Madres adolescentes

Otras madres

Madres adolescentes

Otras madres

Madres adolescentes

Problemas para ser independiente

Otras madres

Madres adolescentes

Atención dirigida sólo a ella

Otras madres

Madres adolescentes

Atención dirigida a otra persona

Otras madres

Madres adolescentes

Accesos frecuentes de furia

Otras madres

Madres adolescentes

Trastorno por estrés post-traumático

Otras madres

Madres adolescentes

Carencia de auto-respeto

Otras madres

Madres adolescentes

Carencia de auto-confianza

Otras madres

Madres adolescentes

Deprimida

Descuido de la higiene

Otras madres

Madres adolescentes

Otras madres

Madres adolescentes

Buena cooperación durante la entrevista

\begin{tabular}{rrcr}
$\mathrm{n}$ & \multicolumn{1}{c}{$\%$} & $\mathrm{Chi}^{2}$ & $\mathrm{I} . \mathrm{C}$. \\
$\mathbf{9 1}$ & $\mathbf{1 4 , 5 8}$ & $\mathbf{5 , 5 3}$ & $\mathbf{9 5 \%}$ \\
$\mathbf{2 8}$ & $\mathbf{2 3 , 1 4}$ & & \\
235 & 37,66 & 1,20 & $95 \%$ \\
52 & 42,98 & & \\
57 & 9,13 & 1,92 & $95 \%$ \\
16 & 13,22 & & \\
$\mathbf{6 2}$ & $\mathbf{9 , 9 4}$ & $\mathbf{4 , 5}$ & $\mathbf{9 5 \%}$ \\
$\mathbf{2 0}$ & $\mathbf{1 6 , 5 3}$ & & \\
12 & 1,92 & 2,22 & $95 \%$ \\
5 & 4,13 & & \\
21 & 3,37 & 0,99 & $95 \%$ \\
2 & 1,65 & & \\
27 & 4,33 & 2,13 & $95 \%$ \\
9 & 7,44 & & \\
59 & 9,46 & 0,19 & $95 \%$ \\
13 & 10,74 & & \\
34 & 5,45 & 2,37 & $95 \%$ \\
11 & 9,09 & & \\
72 & 11,54 & 1,11 & $95 \%$ \\
10 & 8,26 & & \\
$\mathbf{1 1 0}$ & $\mathbf{1 7 , 6 3}$ & $\mathbf{4 , 4}$ & $\mathbf{9 5 \%}$ \\
$\mathbf{1 2}$ & $\mathbf{9 , 9 2}$ & & \\
39 & 6,25 & 2,14 & $95 \%$ \\
12 & 9,92 & & \\
$\mathbf{2 9 6}$ & $\mathbf{4 7 , 4 4}$ & $\mathbf{5 , 0 1}$ & $\mathbf{9 5 \%}$ \\
$\mathbf{4 4}$ & $\mathbf{3 6 , 3 6}$ & & \\
& & &
\end{tabular}

* Las filas en negrita representan diferencias significativas. 
Tabla V

Entendimiento/comprensión del bebé

\begin{tabular}{llrrrr} 
Variable & \multicolumn{1}{c}{ Grupo } & $\mathrm{n}$ & $\%$ & $\mathrm{Chi}^{2}$ & I.C. \\
\hline Saber cuándo empieza a sentarse el niño & Otras madres & 435 & 69,71 & 14,24 & $99 \%$ \\
& Madres adolescentes & 63 & 52,07 & & \\
Saber qué leche es la mejor para el niño & Otras madres & 450 & 72,12 & 8,7 & $99 \%$ \\
& Madres adolescentes & 71 & 58,68 & & \\
Saber cuándo empieza a hablar el niño & Otras madres & 390 & 62,5 & 16,01 & $99 \%$ \\
& Madres adolescentes & 52 & 42,98 & & \\
Saber cuándo empieza a andar el niño & Otras madres & 414 & 66,35 & 10,3 & $99 \%$ \\
& Madres adolescentes & 62 & 51,24 & & \\
El cuidado del niño es una tarea dura & Otras madres & 79 & 12,66 & 6,52 & $95 \%$ \\
& Madres adolescentes & 26 & 21,49 & & \\
Cuando el niño llora, sé por qué & Otras madres & 373 & 59,78 & 9,51 & $99 \%$ \\
& Madres adolescentes & 54 & 44,63 & & \\
Cuando el niño llora, a veces sé por qué & Otras madres & 155 & 24,84 & 4,28 & $95 \%$ \\
& Madres adolescentes & 41 & 33,88 & & \\
Cuando el niño llora, no sé por qué & Otras madres & 42 & 6,73 & 5,95 & $95 \%$ \\
& Madres adolescentes & 16 & 13,22 & & \\
El niño no tiene miedo a personas extrañas & Otras madres & 98 & 15,71 & 4,53 & $95 \%$ \\
& Madres adolescentes & 10 & 8,26 & & \multirow{2}{*}{ El niño no se dirige espontáneamente a ella } \\
& Otras madres & 52 & 8,33 & 7,8 & $99 \%$ \\
& Madres adolescentes & 20 & 16,53 & & \\
El niño llama a la madre mediante gestos & Otras madres & 98 & 15,71 & 1,97 & $95 \%$ \\
& Madres adolescentes & 13 & 10,74 & & \\
\hline
\end{tabular}

Tabla VI

Cuidados del niño*

\begin{tabular}{llrrrr} 
Variable & \multicolumn{1}{c}{ Grupo } & $\mathrm{n}$ & \multicolumn{1}{c}{$\%$} & $\mathrm{Chi}^{2}$ & I.C. \\
\hline Niños superprotegidos & Otras madres & $\mathbf{7 0}$ & $\mathbf{1 1 , 2 2}$ & $\mathbf{4 , 3 3}$ & $\mathbf{9 5 \%}$ \\
Niños normalmente protegidos & Madres adolescentes & $\mathbf{6}$ & $\mathbf{4 , 9 6}$ & & \\
& Otras madres & 509 & 81,57 & 0,87 & $95 \%$ \\
Niños cuidados negligentemente & Madres adolescentes & 103 & 85,12 & & \\
& Otras madres & 23 & 3,69 & 1,69 & $95 \%$ \\
Niños con un cuidado higiénico negligente & Madres adolescentes & 7 & 5,79 & & \\
\cline { 2 - 4 } Infecciones de la piel & Otras madres & $\mathbf{3 5}$ & $\mathbf{5 , 6 1}$ & $\mathbf{4 , 4 3}$ & $\mathbf{9 5 \%}$ \\
& Madres adolescentes & $\mathbf{1 3}$ & $\mathbf{1 0 , 7 4}$ & & \\
Infecciones frecuentes de los órganos & Otras madres & 91 & 14,58 & 3,65 & $95 \%$ \\
respiratorios & Madres adolescentes & 26 & 21,49 & & \\
& Otras madres & $\mathbf{6 5}$ & $\mathbf{1 0 , 4 2}$ & $\mathbf{3 , 7 5}$ & $\mathbf{9 5 \%}$ \\
\hline
\end{tabular}

\footnotetext{
* Las filas en negrita representan diferencias significativas.
} 
nificativamente más frecuente en el grupo de madres adultas.

Las madres adolescentes documentaron con mayor frecuencia falta de conocimientos sobre el desarrollo del bebé y sobre sus necesidades. Los problemas del cuidado del niño y del entendimiento de las necesidades del bebé, tan importantes para una respuesta adecuada (acorde) también estaban presentes, junto con otros problemas, en las madres adolescentes.

La desatención de los niños no fue rara en ambos grupos de madres, y se evidenció por cuidados inadecuados, falta de higiene, abandono, infecciones de la piel, infecciones respiratorias, anemia, raquitismo, problemas digestivos y resultados alterados en la exploración general. Comparando estos dos grupos de madres y sus hijos, encontramos un número significativamente mayor de madres jóvenes negligentes respecto a la higiene de sus hijos, con un número significativamente mayor de infecciones respiratorias. Las enfermeras pediátricas y los pediatras documentaron todos los parámetros citados.

\section{Conclusiones}

Durante la guerra en Croacia y en BosniaHerzegovina, los adolescentes fueron, seguramente, el grupo de población menos protegido y más abandonado. En particular, aquellos adolescentes acomodados en campos de refugiados y centros colectivos, que habían perdido el tiempo en habitaciones llenas de humo, almacenados con ayuda humanitaria y cigarrillos. Crecieron en medio de la incertidumbre y de la pobreza, con perspectivas de futuro poco claras, y sin haber asistido al colegio para nada.

En condiciones tan extremas como el ambiente traumático de guerra, la materni- dad adolescente genera un estrés adicional, que podría tener potenciales consecuencias en el crecimiento de los hijos. El niño podría llegar a ser tratado negligentemente como resultado de semejantes condiciones duras de vida, tanto de la madre adolescente como de la madre adulta.

Además, se requieren medidas adicionales de intervención con métodos estándar de prevención y tratamiento del abandono infantil, bajo las condiciones de vida de los refugiados.

\section{Bibliografía}

ACHENBACH, T.M. Int. J. of Ment. Heal, 7, 24, 1979.

BROOKS-GUNN, J., FURSTENBERG, F.F. Developmental Review, 6, 224, 1986.

DŽEPINA, M., ŠTAMPAR, D. Obilježja spolnog ponašanja adolescenasta, Zbornik radova II Kongres liječnika skolske i univerzitetske medicine, Subotica, 37, 1990.

FURSTENBERG, F.F., Jr. BROOKS GUNN, J. MORGAN, P. Adolescent mothers in later life. (New York: Cambridge University Press 1987).

GARRISON, C.Z., SCHLUCHTER, M.D., SCHOENBACH, V.J., KAPLAN, B.K. Jour. Am. Ac. of Child and Adol. Psych, 28, 341, 1989.

OSOFSKY, D.J., PEEBLES, C., FICK, A., HANN, D.M. Manuscript submitted for publication, 1992.

OSOFSKY, D.J., EBERHART-WRIGHT, A. Int. J. of PsycAn, 69, 221, 1988.

OSOFSKY, J.D. Final report to the Institute of Mental Hygiene, New Orleans, 1991.

ŠTAMPAR, D., BELUHAN, A. Arhiv zaštite majke $i$ djeteta, 35: 189, 1991.

ZURAVIN, S.J. Am. Jour. of Orthopsych, 59, 1989.

Dirección para correspondencia:

Dr. Mladen Knežević

Drage Ivanisevica Za

10000 Zagreb

CROATIA / HRVATSKA 